\title{
Classifying Postherniorrhaphy Pain Syndromes Following Elective Inguinal Hernia Repair
}

\author{
M. J. A. Loos · R. M. H. Roumen · M. R. M. Scheltinga
}

Published online: 14 June 2007

(C) Société Internationale de Chirurgie 2007

\begin{abstract}
Background Chronic postherniorrhaphy pain is diverse in origin. The aim of our study was to classify postherniorrhaphy pain syndromes following elective inguinal hernia repair.

Patients and methods All patients with an elective inguinal hernia repair performed between January 2000 and August 2005 received a questionnaire evaluating chronic inguinal pain (visual analog scale, VAS 0-10). Patients with moderate to severe pain complaints (VAS score $\geq 3$ ) were invited for an interview and an outpatient department physical examination.

Results A total of 2,164 cases underwent an elective hernia repair and received the questionnaire; 1,766 individuals responded (response rate: $81.6 \%$ ). Moderate to severe pain was present in 211 patients (11.9\%). Follow-up was performed in 148 patients. Three separate groups of diagnoses were identified. Group I: neuropathic pain $(n=72)$ indicating inguinal nerve damage; group II: nonneuropathic pain $(n=40)$ due to an array of diagnoses including periostitis $(n=18)$ and recurrent hernia $(n=13)$; and group III: a tender spermatic cord and/or a tight feeling in the lower abdomen $(n=43)$.

Conclusions Chronic pain following elective hernia repair is common and diverse in etiology but may allow for a classification contributing to the development of tailored treatment regimens.
\end{abstract}

This study was presented at the Annual Meeting of the British Hernia Society in Nottingham, November 2006.

M. J. A. Loos $(\square)$ · R. M. H. Roumen

M. R. M. Scheltinga

Department of Surgery, Máxima Medical Centre, PO Box 7777,

De Run 4600, Veldhoven, The Netherlands

e-mail: M.Loos@mmc.nl
Chronic pain following elective inguinal hernia repair is common. Approximately $14 \%-54 \%$ of patients still experience some degree of inguinal pain several years after "successful", surgery [1-6]. Moreover, up to $21 \%$ of patients are functionally impaired in work or leisure activities $[1,2]$. As many as $1 \%$ of individuals suffering from pain after open repair are eventually referred to a specialized pain clinic, as are $0.4 \%$ after laparoscopic hernia repair [7].

Efforts have been made to clarify the etiology of these postoperative pain syndromes using pain descriptors in questionnaires $[2,3]$. In such studies, neuropathic symptomatology was more often described than non-neuropathic descriptors, suggesting a significant nerve-related contribution to pain. However, this result allows for only a limited insight into underlying causes. A complete physical examination, possibly supported by additional testing, may provide answers in the quest for a correct diagnosis and tailored treatment regimens. The aim of the present study was to classify postherniorrhaphy pain syndromes following elective inguinal hernia repair.

\section{Definitions}

Some authors have attempted to classify inguinal pain after hernia repair $[8,9]$. In the present study it was decided to make a distinction between neuropathic and non-neuropathic (nociceptive) causes of pain, as suggested by Amid [9]. Neuropathic pain is characterized as an activity-induced sharp pain, located in proximity to the inguinal scar. The pain frequently radiates toward the scrotum, labium, and/or upper inner thigh. Upper body stretching or twisting and/or hip joint flexing may cause pain from nerve traction or 
compression. Physical examination often reveals signs of a disturbed neurophysiological equilibrium including hypoesthesia, hyperesthesia, or allodynia. A distinct trigger point situated in or close to the scar may cause pain following stimulation, e.g., after palpation. A local anesthetic nerve block can possibly act as a diagnostic and (temporary) therapeutic agent. The complex of symptomatology is thought to be caused by entrapment of ilioinguinal, iliohypogastric, or genital branches of the genitofemoral nerves. Suture material, staples or tacks, perineural fibrosis, and prosthetic material have all been implicated, as has accidental iatrogenic nerve damage, possibly causing a neuroma.

In non-neuropathic causes of inguinal pain after hernia repair, other conditions are responsible for symptomatology including residual/recurrent hernias, hip pathology, and periostitis pubis, among others. In such cases, all nerves are intact. These definitions of the neuropathic and non-neuropathic causes of pain are applicable in the following text.

\section{Patients and Methods}

The study was conducted at the Máxima Medical Centre, a teaching hospital serving approximately 350,000 inhabitants in the Eindhoven and Veldhoven region, the Netherlands. Patients were eligible for study if they reported moderate or severe pain (visual analog scale [VAS] $\geq 3$; range: $0=$ no pain, $10=$ unbearable pain) as identified by a recent questionnaire study (Fig. 1) [1].
Eligible patients were contacted and invited to come to the Surgical Outpatient Department for a standardized interview and physical examination. Current pain intensity was then tested once again, using the VAS-scoring procedure.

Patients received a local injection of $10 \mathrm{cc}$ lidocain $(1 \%)$ if the combination of the patient's history and the physical examination (trigger point) suggested pain of neuropathic origin. If a non-neuropathic origin of pain was suspected, the treatment approach depended on the suggested diagnosis. For instance, if a periostitis was diagnosed, patients received a local injection containing $5 \mathrm{cc}$ lidocaine and $40 \mathrm{mg}$ methylprednisolone acetate $40 \mathrm{mg} / \mathrm{ml}$ (DepoMedrol $\left.{ }^{\circledR}\right)$, a corticosteroid, at the site of maximal pain intensity. Following a 10-min equilibrium period after injection, the regimen's efficacy was evaluated by a VAS score. Additional imaging techniques including ultrasound, computed tomography (CT) scans, or magnetic resonance imaging (MRI) were performed if deemed necessary.

\section{Results}

Demographic and pain characteristics

Figure 1 describes patient inclusion. A total of 211 patients (11.9\%) were eligible for the study, as dictated by a VAS-score $\geq 3$. Sixty-three patients did not visit the outpatient department for reasons stated in Figure 1, leaving 148 patients $(8.4 \%)$ for analysis. The mean age of participants was 40 years, and the majority were male $(87.2 \%$;
Fig. 1 Flow chart of included and excluded patients following an elective inguinal hernia repair

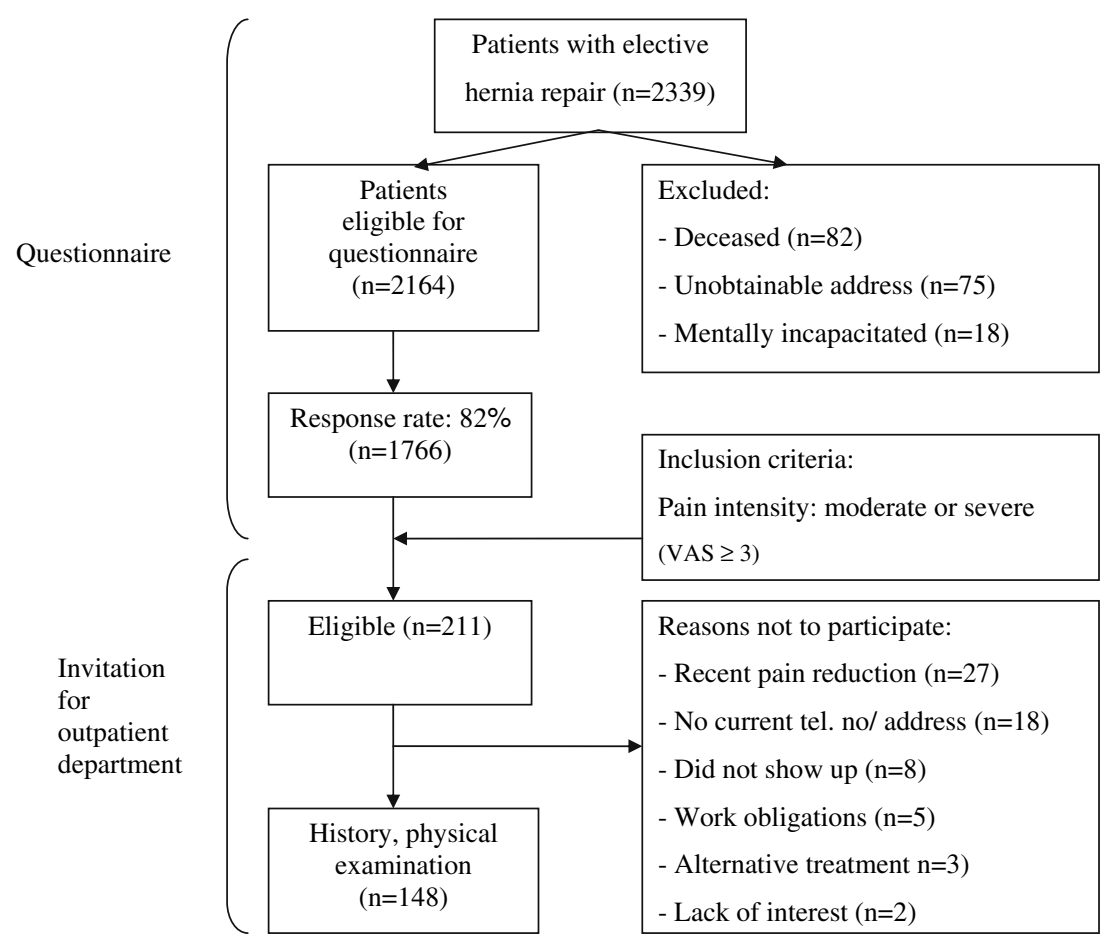


Table 1 Clinical characteristics of patients $(n=148)$ who visited the outpatient department based on a high pain score (VAS $\geq 3$ ) following elective hernia surgery

\begin{tabular}{ll}
\hline & $n(\%)$ \\
\hline Mean age, years [range] & $40[22-69]$ \\
Sex ratio male/female & $129 / 19(87.2 / 12.8)$ \\
Surgical technique & \\
Lichtenstein & $103(69.6)$ \\
Shouldice & $10(6.8)$ \\
TEP & $19(12.8)$ \\
TAPP & $16(10.8)$ \\
Surgery for recurrent hernia & $38(25.7)$ \\
Bilateral hernia repair & $32(21.6)$ \\
Median follow-up, months [range] & $46[3-300]$ \\
\hline
\end{tabular}

Values in parentheses are percentages, unless otherwise specified TEP total extraperitoneal; TAPP transabdominal preperitoneal

Table 1). Most hernia operations were done by open techniques (76.4\%, mainly Lichtenstein), and about one quarter of patients (23.6\%) treated using a laparoscopic method (total extraperitoneal [TEP], 12.8\%; transabdominal preperitoneal [TAPP], 10.8\%).

The pain history of the study population is given in Table 2. Almost $90 \%$ reported groin pain prior to corrective surgery. However, the present postoperative pain level was judged comparable or worse by half of the patients. In almost every case the pain had started directly after surgery, and its severity was considered by 28 patients (18.9\%) to be progressive. More than half of the patients were constantly suffering from pain. Other chronic pain syndromes (chronic headache, low back pain, etc) were reported by $31.8 \%$ of patients.

Overall, 26 male patients (20.2\%) reported a bothersome or even incapacitating sensation during or after ejaculation, which was frequently described as "burning" or "stabbing." One patient mentioned a bothersome feeling of mechanical obstruction during ejaculation. Most of these patients $(16 / 26)$ were contending with neuropathic pain complaints as well. Eighteen men complained of increasing inguinal pain during an erection. Testicular pain was mentioned by 17 patients. Not all patients with testicular pain had ejaculatory complaints, or a painful erection. A direct postoperative onset of impotence was mentioned by 3 patients.

Physical examination

Findings on physical examination are listed in Table 3. Inspection revealed bulges in $8.8 \%$ of patients. Palpation identified a distinct trigger point in or around the scar in the nearly half of patients $(46.6 \%)$. Moreover, the pubic tubercle was painful in $12.2 \%$ of patients. Neurophysiological abnormalities were frequently observed. Hypoesthesia was
Table 2 Pain characteristics of 148 patients who visited the outpatient department for pain following inguinal hernia repair

\begin{tabular}{|c|c|}
\hline Pain characteristics & $n(\%)$ \\
\hline Inguinal pain prior to surgery & $132(89.2)$ \\
\hline Postoperative inguinal pain comparable or worse & $74(50.0)$ \\
\hline Current VAS-score, median $(25 \%-75 \%)$ & $4.0(2.5-5.5)^{\mathrm{a}}$ \\
\hline Time of onset after surgery, median [range] & $0[0-60]$ \\
\hline Duration of pain, median [range] & $31[3-300]$ \\
\hline \multicolumn{2}{|l|}{ Frequency } \\
\hline Occasionally & $18(12.2)$ \\
\hline Regularly & $52(35.1)$ \\
\hline Always & $77(52.0)$ \\
\hline \multicolumn{2}{|l|}{ Course of pain } \\
\hline Constant & $85(57.4)$ \\
\hline Intermittent & $13(8.8)$ \\
\hline Progressive & $28(18.9)$ \\
\hline Decreasing & $21(14.2)$ \\
\hline \multicolumn{2}{|l|}{ Location } \\
\hline Inguinal/pubic region & $134(90.5)$ \\
\hline Scrotum & $17(13.2)$ \\
\hline Labium & $7(36.8)$ \\
\hline Medial thigh & $25(16.9)$ \\
\hline Lower abdomen & $13(8.8)$ \\
\hline \multicolumn{2}{|l|}{ Pain triggers } \\
\hline Erection & $18(13.9)$ \\
\hline Ejaculation & $26(20.2)$ \\
\hline Chronic pain syndromes $^{\mathrm{b}}$ & $47(31.8)$ \\
\hline \multicolumn{2}{|l|}{ Work status } \\
\hline Working & $73(49.3)$ \\
\hline Disabled (workers' compensation) & $40(27.0)$ \\
\hline Retired & $27(18.2)$ \\
\hline None & $7(4.7)$ \\
\hline
\end{tabular}

Values in parentheses are percentages, unless otherwise specified

a VAS-score as measured at outpatient department

b Chronic pain syndromes: chronic headache, back pain, rheumatoid arthritis, fibromyalgia, irritable bowel syndrome

diagnosed in 95 patients, whereas hyperesthesia was present in 11 cases. No patient showed signs of allodynia.

Proposed classification

\section{Group I: neuropathic pain}

A classification including different causes of pain is provided in Table 4. Pain was judged to be neuropathic in nearly half of the patients $(n=72 ; 46.5 \%)$. They all complained of an activity-induced sharp pain combined with a trigger point and signs of a neurophysiological disequilibrium. Eleven patients showed hyperesthesia. All patients were offered a peripheral nerve block with $10 \mathrm{cc}$ lidocaine, and 51 patients 
Table 3 Physical examination and treatment of 148 patients who visited the outpatient department

\begin{tabular}{ll}
\hline Symptom & $n(\%)$ \\
\hline Bulge & $13(8.8)$ \\
Pain pressing pubic tubercle & $18(12.2)$ \\
Trigger point & $69(46.6)$ \\
Neurophysiology & \\
Normal & $42(28.4)$ \\
Hypoesthesia & $95(64.2)$ \\
Hyperesthesia & $11(7.4)$ \\
Nerve block (lidocain) & $51(34.5)$ \\
Significant pain reduction & a \\
Periostal injection (Lidocain/corticosteroids) & 41 \\
Significant pain reduction & $8(5.4)$
\end{tabular}

Values between parentheses are percentages

a Significant pain reduction defined as $>50 \%$ VAS reduction after $10 \min$

Table 4 Classification of chronic inguinal pain in 148 patients after elective inguinal hernia repair

\begin{tabular}{lc}
\hline & $n(\%)$ \\
\hline I Neuropathic cause & $72(46.5)$ \\
Inguinal nerves $^{\mathrm{a}}$ & $71(45.8)$ \\
LFCN (meralgia paresthetica) & $1(0.6)$ \\
II Non-neuropathic cause & $40(25.8)$ \\
Periostitis (pubic tubercle) & $18(11.6)$ \\
Recurrent inguinal hernia & $13(8.4)$ \\
Femoral hernia & $1(0.6)$ \\
Iliopectineal bursitis & $1(0.6)$ \\
Adductor tendinitis & $1(0.6)$ \\
Osteoarthritis of the hip & $1(0.6)$ \\
Referred lumbosacral pain & $3(1.9)$ \\
Urological problems & $2(1.3)$ \\
III Tender spermatic cord/tight feeling & $43(27.7)$ \\
Total number of diagnoses & $155(100)$ \\
\hline
\end{tabular}

Values in parentheses are percentages

Seven patients were diagnosed with two separate conditions

a Ilioinguinal, iliohypogastric, genitofemoral nerve

$L F C N$ lateral femoral cutaneous nerve

agreed to proceed with this treatment. The remainder of the group declined the treatment, 14 patients because they did not consider their pain serious enough, 2 because they had previously received a successful nerve block; 2 because they had contraindications (e.g., bleeding disorders), and 3 because they were afraid of needles. Eighty percent of all patients receiving a local block $(n=41)$ reported pain relief (VAS scores $>50 \%$ lower). Pain relief was not attained in the remaining 10 patients. In one patient meralgia paresthetica was present.

\section{Group II: non-neuropathic pain}

Non-neuropathic causes of pain were detected in forty individuals. In 18 patients a periostitis pubis was diagnosed. On examination their pain was clearly situated on the pubic tubercle, possibly as a result of an incorrectly positioned deep suture. Eight patients with a suspected periostitis received an injection with lidocaine and corticosteroids in tissue overlying the painful periosteum for diagnostic purposes. All eight participants reported pain reduction of more than $50 \%$ on their VAS-score. An injection was refused by 10 patients, because of reasons described in the previous text.

Thirteen recurrences and one femoral hernia were diagnosed, some with the help of an ultrasound or CT scan. Seven patients had a contralateral inguinal hernia as well. In an 18-year-old soccer player bilateral adductor tendinitis was diagnosed. One 45-year-old woman with painful and limited hip endorotation suffered from an iliopectineal bursitis. She regained persistent full pain-free motion of the hip after an intrabursal injection with lidocaine and corticosteroids. Patients with "non-surgical" problems, including hip osteoarthritis, referred lumbosacral pain, and urological problems, were referred to specialists $(n=6)$ who confirmed these diagnoses at a later stage.

\section{Group III: pain possibly related to spermatic cord}

Forty-three patients $(27.7 \%)$ could not be identified on the basis of an existing classification. For the most part, these patients described their pain as "aching" in the absence of a specific trigger point. The spermatic cord was often diffusely tender in those patients who had undergone the hernia repair via an anterior approach. Similarly, in selected cases the mesh inserted during laparoscopic surgery produced a tight aching feeling in the lower abdomen, especially during exercise. In most cases no neurophysiological abnormalities were present. Combining pain history, physical examination, and additional tests, 155 diagnoses could be made in 148 patients. In 7 patients a second cause for the pain was present: periostitis $(n=5)$, hernia recurrence $(n=1)$, and ipsilateral adductor tendinitis $(n=1)$.

\section{Discussion}

The issue of unraveling the dilemma of long-term moderate to severe postherniorrhaphy pain is not new [10]. However, to our knowledge this is the first study in which a large cohort of patients was examined at the outpatient department to clarify the underlying mechanisms responsible for pain following hernia repair. A similar but smaller study was conducted by Cunningham et al. in 1996 [8]. In that 
study a subset of 10 patients referred to a pain clinic with persisting pain was investigated 2 years after inguinal repair. The authors proposed three distinct pain syndromes; somatic $(n=9)$, neuropathic $(n=1)$, and visceral $(n=1)$. The first one was judged as a ligamentous pain syndrome caused by suture insertion in the iliopubic tract and periosteum. The second syndrome was neuralgic and caused by inguinal sensory nerve damage, whereas a third complex was associated with ejaculation pain. They concluded that severe pain syndromes following hernia repair are most commonly somatic in origin. Similar groups of patients were identified in the present study. Pain of neuropathic origin was suspected in nearly half of the patients and was confirmed by nerve block in $28 \%$ of all patients. If one extrapolates these results to the initial patient population encompassing 1,766 individuals, one could cautiously assume that at least $4 \%$ of all corrected inguinal hernias are associated with nerve entrapment or damage. Previous authors have estimated a similar prevalence, varying between $3 \%$ and 5\% [11]. Chronic nerve irritation should be considered an important cause of moderate or severe chronic pain after inguinal hernioplasty.

Another well-known source of postherniorrhaphy pain is periostitis of the pubic tubercle [12]. A too deeply positioned suture aimed at medially affixing the mesh may cause inflammation and chronic irritation. In the examined cohort, one in every eight patients experienced pain while exerting digital pressure on the pubic tubercle. This pain syndrome can be avoided by careful placement of suture material by the surgeon, ideally sparing the bone's periosteal layers. An injection with a local anesthetic and corticosteroids in painful periosteum can be tried as the first line of treatment, as this was effective in a substantial number of our patients. Surgical suture removal must be considered if pain persists.

When a patient presents with residual pain following hernial repair, a recurrent hernia is often the only diagnosis that surgeons consider and rule out. Although relatively infrequent in the present study, 13 patients did have such a recurrence. This number approximates the $1 \%$ of the initial 1,766 patients used in our previous questionnaire study. The recurrence rate is probably higher because asymptomatic and mildly symptomatic recurrences remain undetected.

A variety of additional musculoskeletal problems were observed in the remainder of the patients with recurrent pain, including iliopectineal bursitis, adductor tendinitis, and referred low back pain. These pain syndromes are very likely the secondary result of postural and functional changes in the presence of persisting inguinal pain. However, a third group of 43 individuals demonstrated a clearly distinct history and physical examination. They presented with a tender spermatic cord (after open mesh repair) or a tight aching feeling in the lower abdomen (after laparoscopic procedures). Compression by scar tissue or prosthetic material may explain this type of groin pain [9]. Compromised musculotendinous structures might play a roll as well. It remains unclear if venous congestion contributes to pain in this group of patients. Overall pain intensity is less pronounced than that reported by the neuropathic pain group, although most patients experienced some limitation in daily activities. Except for pain medication, no treatment was available for them. We suggest naming this type of pain "funiculodynia,', as this syndrome is mainly characterized by pain in structures surrounding the spermatic cord.

Prevalence, etiology, and treatment of genital complications following hernia repair including erectile and ejaculatory pain are largely unknown. In a recently published Danish questionnaire study 3\% of younger male patients with inguinal hernia repair exhibited pain during sexual activity and subsequent sexual dysfunction [13]. In the present study dysejaculation was reported by one of every five male patients. Several pathophysiological mechanisms have been suggested, among them intraoperative nerve damage, dysfunction of periurethral structures involved in ejaculation, or encasement of the spermatic cord caused by scar tissue. This is supported by anecdotal reports on patients with dysejaculation in which dissection of twisted fibrotic spermatic cords combined with an ilioinguinal neurectomy provided total pain relief [14]. Because of the high incidence of such complaints and the sparse literature, more research on the etiology and treatment of dysejaculatory conditions after inguinal hernioplasty is definitely warranted.

Over $30 \%$ of all patients reported suffering from other chronic pain syndromes as well. A correlation between the onset of postherniorrhaphy pain and other pain syndromes has been described in the hernia literature, and it may be due to genetic and psychosocial factors [13]. Patients with a tendency to develop chronic pain are more susceptible to develop additional pain syndromes.

Classifying postherniorrhaphy pain syndromes may allow for tailored treatment regimens. The first step in a protocol for treatment of postherniorrhaphy neuralgia, described by Lichtenstein nearly two decades ago, consisted of primary diagnosis and treatment by injections [10]. Surprisingly, in the present study a single diagnostic nerve block with lidocaine led to long-term ( $>1$ month) pain reduction in $25 \%$ of our patients, confirming the therapeutic potential of such injections. Although it is known to occur, the phenomenon of permanent or long-term cure following injection of short acting anesthetics is not well understood [15]. If (repeated) injection therapy fails, the second step might be operative transsection [10]. After early reports, it was suggested by Amid that transsection should include all three groin nerves, and the procedure was named "triple neurectomy" [9]. 
Because of central and peripheral communication and possible involvement of all three nerves, a maximal length of ilioinguinal, iliohypogastric, and genitofemoral in both directions should be transsected and removed.

Reports on the effect of the mesh on nerves and chronic pain in hernia repair are scarce. According to a recent animal study, inserted mesh may lead to an inflammatory and fibroblastic response resulting in adhesions and mechanical entrapment of adjacent nerve fibers and structures such as the spermatic cord [16]. Whether these mesh-related nerve changes are responsible for any pain sensation is unknown. One study comparing mesh with suturing techniques using the body's own tissue showed similar rates of chronic pain [17]. Nevertheless, removal of mesh in combination with neurectomy appeared to be successful in $60 \%$ of patients with chronic inguinodynia [18]. Apart from its inflammatory potential, implanted mesh may also exert mechanical pressure on neighboring structures or may fold or wrinkle ("meshoma"), causing chronic pain [19]. A (partial) removal of mesh in combination with a (triple) neurectomy may be considered the preferred treatment in patients with severe neuropathic pain in the presence of a meshoma. In concert with a recent review on surgical management of chronic pain after groin hernia repair, there is an obvious need for more prospective research [20].

The results of the present study demonstrate that the differential diagnosis of moderate or severe postherniorrhaphy pain is diverse, but the findings allow for symptom classification with resultant treatment options. Proper research concerning different types of therapy for chronic pain after groin hernia repair is warranted. A randomized controlled trial comparing peripheral injections with neurectomy has recently been initiated by our group of investigators.

\section{References}

1. Loos MJA, Roumen RMH, Scheltinga MRM. (2007) Chronic sequelae of common elective groin hernia repair. Hernia. Epub ahead of publication, Feb 6

2. Bay-Nielsen M, Perkins FM, Kehlet H. (2001) Pain and functional impairment 1 year after inguinal herniorraphy: a nationwide questionnaire study. Ann Surg 233:1-7
3. Nienhuijs SW, Boelens O, Strobbe LJA. (2005) Pain after anterior hernia repair. J Am Coll Surg 200:885-889

4. Callesen T, Bech K, Kehlet H. (1999) Prospective study of chronic pain after groin hernia repair. Br J Surg 86:1528-1531

5. Poobalan AS, Bruce J, King PM, et al. (2000) Chronic pain and quality of life following open inguinal hernia repair. Br J Surg 88:1122-1126

6. Page B, Paterson D, Young D, et al. (2002) Pain from primary inguinal hernia and the effect of repair on pain. $\mathrm{Br} \mathrm{J}$ Surg 89:1315-1318

7. Hindmarch AC, Cheong E, Lewis MPN, et al. (2003) Attendance at a pain clinic with severe chronic pain after open and laparoscopic inguinal hernia repairs. Br J Surg 90:1152-1154

8. Cunningham J, Temple WJ, Mitchell P, et al. (1996) Cooperative hernia study: pain in the postrepair patient. Ann Surg 224:598602

9. Amid PK. (2004) Causes, prevention, and surgical treatment of postherniorrhaphy neuropathic inguinodynia: Triple neurectomy with proximal end implantation. Hernia 8:343-349

10. Lichtenstein IL, Schulman AG, Amid PK. (1988) Cause and prevention of postherniorrhaphy neuralgia: A proposed protocol for treatment. Am J Surg 155:786-790

11. Starling JR, Harms BA, Schroeder ME, et al. (1989) Diagnosis and treatment of genitofemoral and ilioinguinal neuralgia. World J Surg 13:586-591

12. Shulman AG. Changes in technique of primary inguinal hernioplasty since 1984. In The Lichtenstein Hernia Repairs, How to Do Them Right! $1^{\text {st }}$ Edition, Wagner Design, ISBN 0-9653526-09,1996;49

13. Aasvang E, Møhl B, Bay-Nielsen M, et al. (2006) Pain related sexual dysfunction after inguinal herniorrhaphy. Pain 122:258 263

14. Butler JD, Hershman MJ, Leach A. (1998) Painful ejaculation after inguinal hernia repair. J R Soc Med 91:432-433

15. Hahn L. (1989) Clinical findings and results of operative treatment in ilioinguinal nerve entrapment syndrome. $\mathrm{Br} \mathrm{J}$ Obstet Gynecol 96:1080-1083

16. Demirer S, Kepenekci I, Evirgen O, et al. (2005) The effect of polypropylene mesh on ilioinguinal nerve in open mesh repair of groin hernia. J Surg Res 131:175-181

17. Vrijland WW, van den Tol MP, Luijendijk RW, et al. (2002) Randomized clinical trial of non-mesh versus mesh repair of primary inguinal hernia. Br J Surg 89:293-297

18. Heise CP, Starling JR. (1998) Mesh inguinodynia: a new clinical syndrome after inguinal herniorrhaphy? J Am Coll Surg 187:514518

19. Amid P. (2004) Radiologic images of meshoma. Arch Surg 139:1297-1298

20. Aasvang E, Kehlet H. (2005) Surgical management of chronic pain after inguinal hernia repair. Br J Surg 92:795-801 